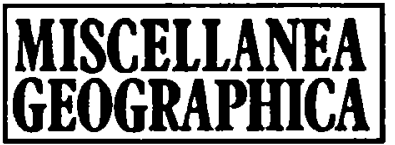

WARSZAWA 1996 Vol. 7

Witold Kusiński

\title{
LE CENTRE ET LA PÉRIPHÉRIE DES GRANDES VILLES EN PÉRIODE DE TRANSITION
}

Dans les pays démocratiques et dans les conditions propres à l'économie de marché libre, le rôle de premier plan revient à l'autoorganisation et à l'autodéveloppement, tandis que celui des autorités centrales aussi bien administratives que politiques se borne, le plus souvent, à inspirer et à régler. L'autoorganisation et l'autodéveloppement, les deux compris comme une façon dont les systèmes complexes passent d'une structure à une autre, d'un ordre à un autre, en résultat d'une action des forces immanentes à un système donné, se fait par une extension de l'étendue, de l'échelle et de l'intensité des rapports fonctionnels existant entre des cités particulières et le centre, ainsi que par un renforcement des relations entre des cités appartenant à un système d'habitat donné et les éléments situés dans d'autres régions ce qui se manifeste par un transfert de personnes et de biens et une transmission d'informations.

Dans les pays où le pouvoir était centralisé et hiérarchisé, la gestion de l'économie était réalisée au moyen des décisions administratives, ces dernières, tout comme les décisions concernant d'autres domaines, étaient prises à l'échelle de l'État, avec lenteur et souvent incompétence, sans parler du haut degré d'idéologisation de la vie sociale et, surtout, de la domination de la politique sur l'économie et d'autres domaines d'activités. Cet état de choses devait nécessairement freiner les processus d'autoorganisation et d'autodéveloppement, ce qui, à son tour, limitait les tentatives visant le déplacement de certains organismes et entreprises du centre vers la périphérie. Le système centralisé du pouvoir et des activités socioéconomiques favorisait une domination du centre sur la périphérie.

Le développement des villes - centres des régions urbaines s'accomplissait au moyen d'une cumulation d'entreprises et d'institutions dont le nombre ne cessait de croître et, ce qui s'ensuit, par un accroissement rapide du nombre d'habitants. La forme spatiale des villes - centres des régions urbaines - faisait penser à une étoile car leur extension allait le long des principales voies d'accès. Tout comme dans les conditions propres à l'économie de marché libre, le long de ces voies se formaient des axes (bandes) de développement. Hors du centre et des axes de développement for- 
mant un ensemble fonctionnel appelé par les géographes russes l'ossature portante" du système d'habitat, se trouvait la périphérie qui y était liée par des liens multiples de dépendance. Il sied de souligner ici que parfois des espaces de diverses grandeurs, qu'on pouvait appeler marginaux, restaient en dehors de ces ensembles. Les espaces en question ou bien n'entraient pas dans la sphère d'influences de centres urbains importants ou encore c'est là que se croisaient de faibles influences de deux ou même plusiers centres urbains. Les possibilités de développement de ces espaces étant limitées, ceux-ci perdaient leur potentiel aussi bien humain qu'économique. L'élimination de tous les vices et défauts structurels, organisationnels et fonctionnels bien qu'initiée par l'État et jouissant de son appui organisationnel et financier, reste l'affaire des collectivités locales. L'État ne doit pas se manifester en tant que force omnipotente - il doit initier et coordonner les transformations. Cette obligation de recourir à l'État résulte d'une pénurie de moyens financiers privés suffisants, indispensables à réaliser les transformations, de la nécessité de réorienter les attitudes d'une grande partie de la collectivité, de l'absence d'une force capable de niveler les tensions et les conflits sociaux, enfin de la nécessité d'adopter de nouvelles normes juridiques.

Les changements socio-économiques visant le développement du marché, réalisés à l'aide de l'adaptation des échanges et de la production aux conditions de la privatisation, ensuite le développement d'un réseau bancaire et de différentes branches d'une infrastructure spéciale (consulting, etc.) se manifestent de façon plus ou moins nette dans les transformations spatiales des structures d'habitat. Les transformations en question sont visibles dans les migrations des populations et les changements de structures de celles-ci, dans les zones et formes de la construction individuelle en plein essor (la construction publique financée par les organismes d'État, les organismes communaux et les coopératives d'habitation a été sensiblement limitée), dans le développement de la motorisation et celui des services. A côté des changements positifs, on note l'apparition des phénomènes appartenant à la pathologie sociale, et notamment le chômage, la drogue, le banditisme, le crime organisé, etc.

En ce qui concerne les études portant sur la relation entre le centre et la périphérie, on voit la nécessité d'étudier les transformations en cours. Les recherches devraient se concentrer sur les groupes suivants de problèmes:

1. Les transformations démographiques dans les villes et dans les zones d'influence de grandes villes, et notamment:

a) le changement de la répartition de la population - migrations vers les quartiers périphériques et la zone suburbaine; l'évolution de l'état de peuplement dans la zone suburbaine et dans les terrains situés à une certaine distance de la ville;

b) les changements de structure biologique - vieillissement croissant de la population, féminisation (ou masculinisation) des collectivités locales; 
c) les changements de la portée et de l'intensité des migrations quotidiennes des travailleurs.

2. Les transformations de la situation matérielle de la population habitant aussi bien le centre que la périphérie, et surtout:

a) le changement des sources des revenus;

b) le changement de l'activité professionnelle et l'intensification du chômage;

c) l'extension des zones de pauvreté.

3. Les transformations des conditions d'habitation, et notamment:

a) le développement de la construction individuelle;

b) le développement des zones et des couloirs (bandes) de cités-satellites dans les espaces voisins de grandes villes.

4. Les transformations dans le domaine des transports, et notamment:

a) les changements des possibilités d'accès du centre de la ville, des centres de régions urbaines ainsi que des zones périphériques;

b) le développement de la motorisations individuelle et les changements dans les transports publics;

c) les changements de la densité du réseau de routes à revêtement dur et amélioré;

d) le développement des services liés avec l'automobile (ateliers de réparation, garages, pompes à essence, points de vente des pièces de rechange) et les changements concernant la localisation de ces services.

5. Les transformations dans le commerce, et notamment:

a) l'accroissement du nombre d'entreprises commerciales (gros, demigros, detail) et les changements concernant leurs localisations respectives;

b) le changement de la structure du commerce;

c) l'installation des "grandes surfaces" à la périphérie des villes ou à une certaine distance de celles-ci.

6. Les transformations dans la sphère de la production et les services de production, et notamment:

a) les transformations relatives à la propriété dans l'industrie (privatisation) et leur influence sur l'emploi et la production;

b) le développement des services de production (développement de l'artisanat productif) et leur localisation.

7. L'évolution du caractère multifonctionnel des terrains agricoles à la périphérie des grandes villes.

En dépit de l'élimination des prémisses formelles et techniques d'une centralisation de différents domaines de la vie, les différences entre le centre et la périphérie ne seront pas nivelées pour autant. Les grandes villes resteront les foyers de la vie sociale, économique, culturelle et politique. Quant à la périphérie, elle aura demeuré un terrain d'influences des centres et, en même temps, le terrain où ces centres iront chercher des biens, des personnes et une énergie nécessaires à leurs activités. Nous ne pouvons que supposer qu'à mesure du développement des transformations socio-politi- 
ques, les prémisses de développement se "normaliseraient", l'auto-organisation et l'autodéveloppement ainsi que la cohérence des régions urbaines ne cesseraient de se renforcer.

Étant donné que les phénomènes de l'autoorganisation et de l'autodéveloppement présenteraient une importante différentiation spatiale, les conditions qui se trouvent à l'origine de cette différentiation devraient faire l'objet d'études approfondies. 